\title{
Generation of high-fidelity four-photon cluster state and quantum-domain demonstration of one-way quantum computing
}

\author{
Yuuki Tokunaga, $, 1,2,3$, 6 Shin Kuwashiro, ${ }^{1,3}$ Takashi Yamamoto, ${ }^{1,3}$ Masato Koashi, ${ }^{1,3}$ and Nobuyuki Imoto ${ }^{1,3}$ \\ ${ }^{1}$ Division of Materials Physics, Graduate school of Engineering Science, \\ Osaka University, Toyonaka, Osaka 560-8531, Japan \\ ${ }^{2}$ NTT Information Sharing Platform Laboratories, NTT Corporation, \\ 3-9-11 Midori-cho, Musashino, Tokyo 180-8585, Japan \\ ${ }^{3}$ CREST Photonic Quantum Information Project, \\ 4-1-8 Honmachi, Kawaguchi, Saitama 331-0012, Japan
}

(Dated: October 24, 2018)

\begin{abstract}
We experimentally demonstrate a simple scheme for generating a four-photon entangled cluster state with fidelity over $0.860 \pm 0.015$. We show that the fidelity is high enough to guarantee that the produced state is distinguished from GHZ, $W$, and Dicke types of genuine four-qubit entanglement. We also demonstrate basic operations of one-way quantum computing using the produced state and show that the output state fidelities surpass classical bounds, which indicates that the entanglement in the produced state essentially contributes to the quantum operation.
\end{abstract}

PACS numbers: 03.67.Mn, 03.65.Ud, 03.67.Lx, 03.67.Hk

There has been much interest in special multi-partite entangled states, called cluster states, because they are used as a resource for one-way quantum computing (QC), in which computation proceeds by a sequence of singlequbit measurements with classical feedforward [1]. Recently, several schemes for preparing cluster states were

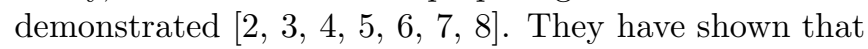
the produced states have genuine multi-partite entanglement and/or given the proof-of-principle demonstration of one-way QC.

In this work, we report an experimental demonstration of a simple scheme for preparing a four-photon cluster state

$$
\begin{aligned}
\left|C_{4}\right\rangle= & \frac{1}{2}\left(|H\rangle_{1}|H\rangle_{2}|H\rangle_{3}|H\rangle_{4}+|H\rangle_{1}|H\rangle_{2}|V\rangle_{3}|V\rangle_{4}\right. \\
& \left.+|V\rangle_{1}|V\rangle_{2}|H\rangle_{3}|H\rangle_{4}-|V\rangle_{1}|V\rangle_{2}|V\rangle_{3}|V\rangle_{4}\right) .
\end{aligned}
$$

Here, $|H\rangle(|V\rangle)$ represents the state of a photon with horizontal (vertical) polarization. The state fidelity of the produced state was over $0.860 \pm 0.015$. This guarantees that not only the produced state has genuine four-qubit entanglement, but also the state is distinguished from classes of genuine four-qubit entangled states including GHZ, $W$, and Dicke types of entangled states. In order to distinguish the produced state from four-qubit Dicke states, the state fidelity should be over $0.75[9]$, which was not achieved in previous four-photon experiments $[2,3,4,5]$. Using the high-fidelity cluster states, we also demonstrated basic operations of one-way quantum computing and obtained high fidelities for output states. Existing demonstrations of one-way QC [2, 3, ,5] gave the output state fidelities of quantum operations only as numeric data. We further evaluate whether the high fidelities of the output states really come from the existence of entanglement of cluster states or not. For that purpose, we propose a classical bound on the fidelity as a solid benchmark for entanglement-based quantum information processing. Then, we show that our experimental results of the basic operations of one-way QC surpass the classical bounds, which indicates that the entanglement of cluster states really contributes to one-way QC. The benchmark can be generally useful for one-way QC and other kinds of experiments of quantum information processing.

Our scheme for preparing $\left|C_{4}\right\rangle$ (Fig. 1) is a slight modification of the scheme for preparing $|\chi\rangle=\frac{1}{2}[(|H H\rangle+$ $|V V\rangle)|H H\rangle+(|H V\rangle+|V H\rangle)|V V\rangle]$ in [10], which is a resource for teleportation-based controlled-NOT gate [11]. Here, $|H\rangle(|V\rangle)$ represents the state of a photon with horizontal (vertical) polarization. Our scheme has fewer requirements and/or a greater success probability compared to the schemes for existing four-photon experiments [2, 3, 4, [5]. It is constructed from four photons produced by parametric down-conversion (PDC), polarizing beam splitters (PBSs), half-wave plates (HWPs), and conventional photon detectors. It does not need polarization dependent beam splitters [3], nor the subwavelength stability of the optical paths [2, 4, 5].

We use spontaneous parametric down-conversion for the preparation of the entangled photon pair [12] and two single photons (Fig. 2). Ultraviolet pulse with a central wavelength of $395 \mathrm{~nm}$, an average power of $220 \mathrm{~mW}$ from a frequency-doubled mode-locked Ti:sapphire laser (wavelength, 790nm; pulse width, $140 \mathrm{fs}$; repetition rate, $76 \mathrm{MHz}$ ) pumps a pair of $1 \mathrm{~mm}$-thick BBO ( $\beta$-Barium Borate, type-I) crystal for PDC. The group delay is compensated by thick quartz crystals $(12.8 \mathrm{~mm})$ to erase the information on the origin (the first or the second $\mathrm{BBO}$ ) of the photon pairs. The relative phase between $H$ and $V$ polarizations is adjusted by a pair of thin quartz crystals $(0.6 \mathrm{~mm})$. The typical two-fold coincidence rate of entangled photon pairs is around $2500 / \mathrm{s}$ and the visibility 


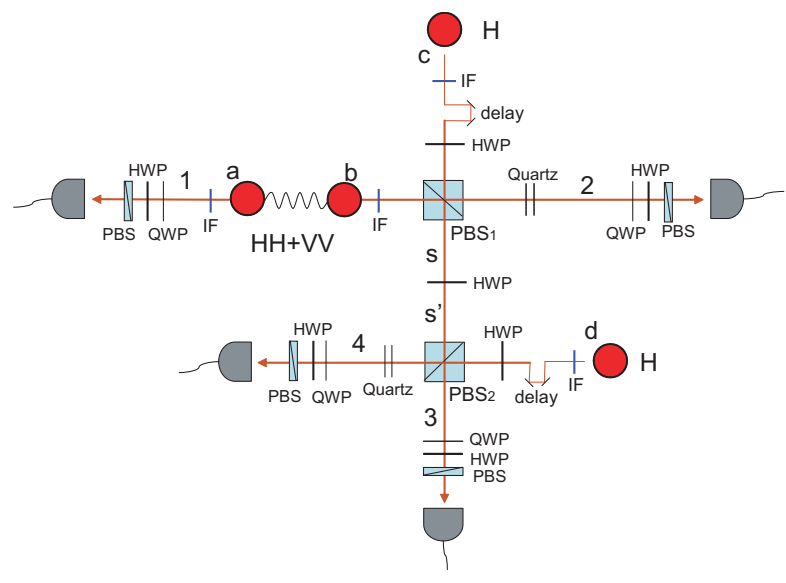

FIG. 1: Experimental setup for preparing $\left|C_{4}\right\rangle$.

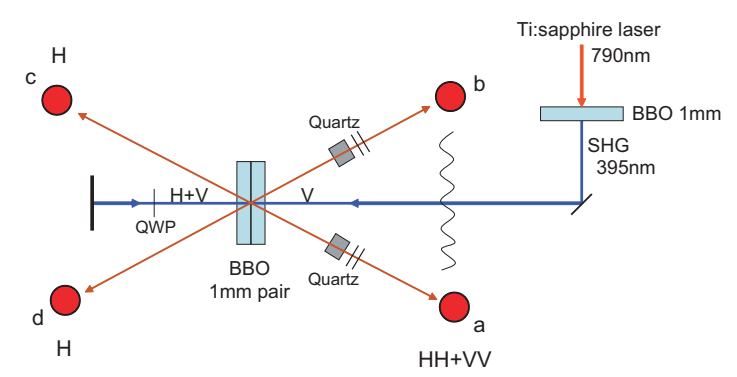

FIG. 2: Experimental setup for preparing two single photons and an entangled photon pair.

is $\sim 97 \%$. The temporal overlap is adjusted by moving mirrors on motorized stages (delays in Fig. 11). The thin quartz crystal pairs in modes 2 and 4 are placed to compensate additional phase shifts. The spectral filtering is achieved with narrow bandwidth interference filters (IFs) with bandwidth of $2.7 \mathrm{~nm}$ (FWHM). Photon detectors (silicon avalanche photodiodes) are placed after single-mode optical fibers to select a single spatial mode to ensure a high visibility. Polarization correlations are recorded by coincidence counting among four photon detectors for various angles of quarter-wave plates (QWPs) and HWPs. The typical four-fold coincidence rate is around 100 per hour.

We obtain lower bounds on the fidelity $F$ of the produced state using methods with fewer measurement settings [9, 13, 14] compared to the method for obtaining the exact fidelity. We denote $X, Y$, and $Z$ for Pauli matrices $\sigma_{x}, \sigma_{y}, \sigma_{z}$, respectively. When a self-adjoint operator $B$ satisfies

$$
\left|C_{4}\right\rangle\left\langle C_{4}\right| \geq B
$$

we can obtain a lower bound on the fidelity by $F \equiv$

$$
\begin{aligned}
\operatorname{Tr}\left[\left|C_{4}\right\rangle\left\langle C_{4}\right| \rho\right] \geq\langle B\rangle \equiv \operatorname{Tr}[B \rho][9] . \text { The operators } \\
B_{2}:=\frac{1}{4}(Z Z I I+I Z X X+Z I X X \\
\quad+X X Z I+I I Z Z+X X I Z)-\frac{1}{2} 11
\end{aligned}
$$

and

$$
\begin{aligned}
& B_{4}:=\frac{1}{8}( X X Z I+I Z X X+Z I X X+X X I Z \\
&-Y Y Z I-I Z Y Y-Z I Y Y-Y Y I Z)
\end{aligned}
$$

satisfy Eq. (2). Therefore, we can obtain lower bounds on the fidelity by measuring expectation values $\left\langle B_{2}\right\rangle$ or $\left\langle B_{4}\right\rangle$. We need two measurement settings $X X Z Z$ and $Z Z X X$ for $B_{2}$ [13, 14], and four measurement settings $X X Z Z, Z Z X X, Y Y Z Z$, and $Z Z Y Y$ for $B_{4}$ [9]. We can obtain a higher lower bound of the fidelity using $B_{4}$.

Figure 3(a) - 3(d) show the 16 possible fourfold coincidence probabilities for measurement settings $X X Z Z$, $Z Z X X, Y Y Z Z$, and $Z Z Y Y$, respectively. Here we denote $| \pm\rangle \equiv \frac{1}{\sqrt{2}}(|H\rangle \pm|V\rangle)$, and $|R / L\rangle \equiv \frac{1}{\sqrt{2}}(|H\rangle \pm i|V\rangle)$. Figure 3 (a') - $3\left(d^{\prime}\right)$ show the corresponding coincidence probabilities for the ideal pure four-photon cluster state $\left|C_{4}\right\rangle$. The error bars are determined by assuming Poissonian counting statistics. Deviation from the ideal case is mainly due to imperfection of indistinguishability of photons and multiphoton emission events with five or more photons. From the four-fold coincidence probabilities in Fig. 3(a) and 3(b), we can calculate $\left\langle B_{2}\right\rangle$ to obtain $F \geq \operatorname{Tr}\left[B_{2} \rho\right]=0.791 \pm 0.030$. If we use all the data in Fig. 3(a) - 3(d) for the four settings, we obtain a much higher bound $F \geq \operatorname{Tr}\left[B_{4} \rho\right]=0.860 \pm 0.015$.

The observed fidelity $F>1 / 2$ assures that the produced state has genuine four-qubit entanglement. Moreover, the high fidelity enables us to discriminate it against several types of genuine four-qubit entangled states. In Ref. 9], it was shown that we can discriminate classes of genuine four-qubit entanglement by extending Schmidt number witness [15] to multiqubit systems. Here we sketch the main idea of the discrimination method. Consider the three ways of partitioning the four qubits 1 , 2,3 , and 4 into two pairs of qubits, (12)(34), (13)(24), and $(14)(23)$. For simplicity, we denote them as 12, 13, and 14, respectively. A pure state of the four qubits can be regarded as a bipartite state for partition $1 j$, and let $r_{1 j}$ be its Schmidt rank. Since the Schmidt rank never increases under local operations and classical communication even probabilistically, the set of the ranks $\left(r_{12}, r_{13}, r_{14}\right)$ is a signature of the state that can be regarded as a (crude) measure of entanglement. The cluster state $\left|C_{4}\right\rangle$ has the signature $(2,4,4)$, while the four-qubit GHZ state $|G H Z\rangle=\frac{1}{\sqrt{2}}(|0000\rangle+|1111\rangle)$ and the $W$ state $|W\rangle=\frac{1}{2}(|0001\rangle+|0010\rangle+|0100\rangle+|1000\rangle)$ both have $(2,2,2)$. The difference can be detected via the fidelity as follows. It was shown [9] that for any state $\left|\eta_{2}\right\rangle$ 

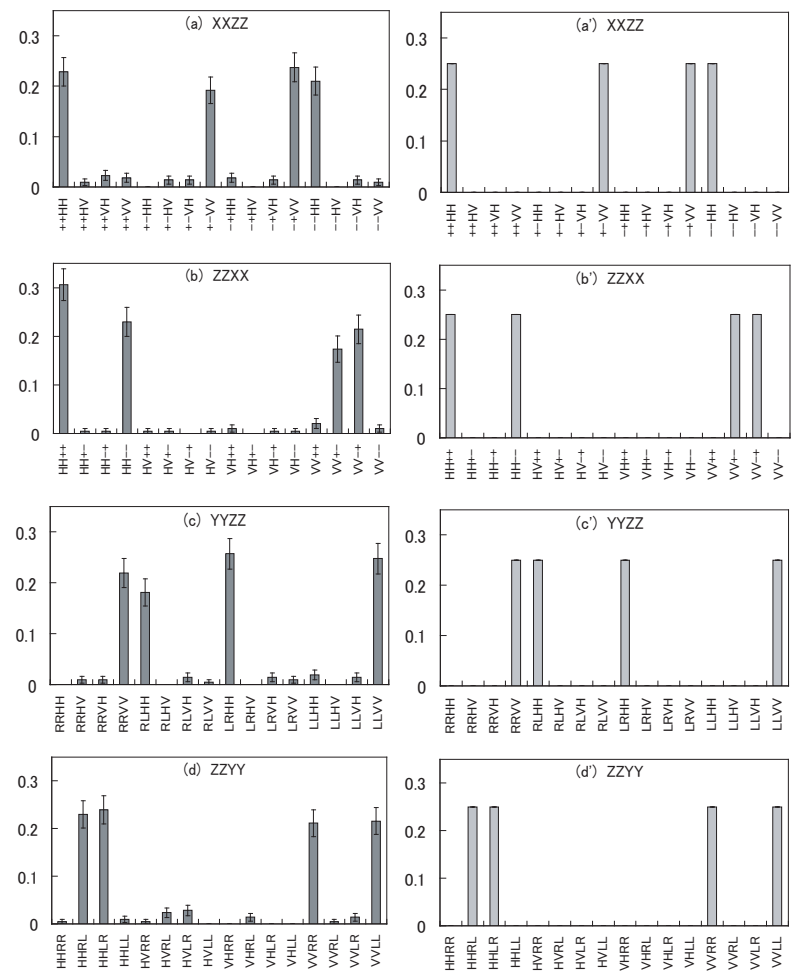

FIG. 3: Four-fold coincidence probabilities for measurement settings (a) $X X Z Z$, (b) $Z Z X X$, (c) $Y Y Z Z$, and (d) $Z Z Y Y$. The ideal cases are shown in $\left(a^{\prime}\right)-\left(d^{\prime}\right)$.

with $r_{13} \leq 2$ or $r_{14} \leq 2$, its fidelity to the cluster state $\left|\left\langle\eta_{2} \mid C_{4}\right\rangle\right|^{2}$ is not greater than $1 / 2$. Hence the observed fidelity of $F>1 / 2$ assures that the produced state is never written as a mixture of states with $r_{13} \leq 2$ or $r_{14} \leq 2$, including the GHZ states and the $W$ states. Similarly, any state with $r_{13} \leq 3$ or $r_{14} \leq 3$ has fidelity not greater than $3 / 4$, and hence $F>3 / 4$ assures that the produced state is never written as a mixture of states with $r_{13} \leq 3$ or $r_{14} \leq 3$, including a four-qubit Dicke state $\left|D_{4}\right\rangle=$ $\frac{1}{\sqrt{6}}(|0011\rangle+|0101\rangle+|0110\rangle+|1001\rangle+|1010\rangle+|1100\rangle)$ having the signature $(3,3,3)$. The experimentally obtained fidelity, $F \geq 0.860 \pm 0.015$, thus discriminates the produced state from the classes of entangled states with the Schmidt rank less than 4 in partition 13 or 14, which include GHZ, $W$, and Dicke types of entangled states.

Next, we report demonstration of basic operations of one-way quantum computing using the produced fourphoton cluster state. What we try to demonstrate here is that the entanglement in the produced four-photon state really contributes to basic operations of one-way QC. In one-way QC, the entanglement in the cluster state enables us to obtain the correct output states with the help of the classical feedforward communication. If it were not for the quantum entanglement, it would be impossible to achieve the correct output states for many kinds of gate instructions with the same amount of classical
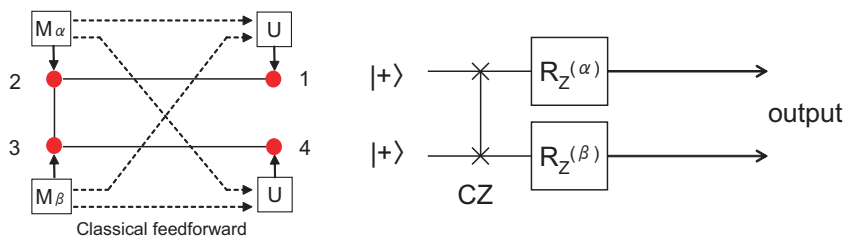

FIG. 4: Physical implementation and the quantum circuit of a two-qubit gate.

communication. This leads to a classical bound on the average fidelity when no entanglement exists between input qubits (for gate instruction) and output qubits. In the following, we first explain the implementation of basic operations in one-way QC. Then we introduce the classical bounds and show that the experimental results are beyond the classical bounds.

Two-qubit gates. We implement the quantum circuit in Fig. 4 via one-way quantum computing model using $\left|C_{4}\right\rangle$. This implementation is basically the same as [2, 5]. The input state is $\left|\psi_{\text {in }}\right\rangle=|+\rangle|+\rangle$. Qubits 2 and 3 are measured in the basis $B(\alpha)$ and $B(\beta)$, where $B(\theta)=\left\{\frac{|0\rangle+e^{-i \theta}|1\rangle}{\sqrt{2}}, \frac{|0\rangle-e^{-i \theta}|1\rangle}{\sqrt{2}}\right\}$. Here we take $\{|0\rangle \equiv|H\rangle,|1\rangle \equiv|V\rangle\}$ as a standard basis. The outcomes are feedforwarded and Pauli operations are applied on qubits 1 and 4 accordingly, resulting in the output state $\left|\psi_{\text {out }}\right\rangle=\left(R_{Z}(\alpha) \otimes R_{Z}(\beta)\right) \mathrm{CZ}\left|\psi_{\text {in }}\right\rangle$ on qubits 1 and 4 . Here, $R_{Z}(\theta)=\exp \left(-i \theta \sigma_{Z} / 2\right)$ and $\mathrm{CZ}$ operation is defined as $|j\rangle|k\rangle \mapsto(-1)^{j k}|j\rangle|k\rangle$, where $j, k \in 0,1$. Note that the gate instructions $(\alpha, \beta)$ are given to qubits 2 and 3 , and only two bits are communicated to qubits 1 and 4. As in the remote state preparation (RSP) protocols [16], the entanglement in the cluster state enables us to obtain the correct output states with the help of the two-bit communication. If it were not for the entanglement, achieving the correct output states for all values of $(\alpha, \beta)$ would be impossible, leading to a bound on the average fidelity. In experiment, we chose 8 combinations of $(\alpha, \beta)$, and measured the fidelity of the output states (the feed-forwarded Pauli operations are substituted by the appropriate reassignment of measurement bases for qubits 1 and 4). Table【 shows the results. Let us determine the upper bound on the average fidelity when we do not have entanglement at all. The only clue about which of the 8 operations are chosen at qubits 2 and 3 is the two-bit signal sent to qubits 1 and 4 . Hence a possible strategy is to divide the 8 states into 4 groups, e.g., (i) $\left|\psi_{1}\right\rangle,\left|\psi_{2}\right\rangle$, (ii) $\left|\psi_{3}\right\rangle,\left|\psi_{4}\right\rangle$, (iii) $\left|\psi_{5}\right\rangle,\left|\psi_{6}\right\rangle$, and (iv) $\left|\psi_{7}\right\rangle$, $\left|\psi_{8}\right\rangle$, and to send the identity of the group. Using this information, qubits 1 and 4 are prepared in one of the states (i) $\left|\psi_{1}\right\rangle+\left|\psi_{2}\right\rangle$, (ii) $\left|\psi_{3}\right\rangle+\left|\psi_{4}\right\rangle$, (iii) $\left|\psi_{5}\right\rangle+\left|\psi_{6}\right\rangle$, and (iv) $\left|\psi_{7}\right\rangle+\left|\psi_{8}\right\rangle$, which were chosen such that the best average fidelity is achieved for each group (normalization factors omitted). This particular strategy gives the average fidelity of the 8 states $\cos ^{2}(\pi / 8) \approx 0.854$. Since the 
TABLE I: Output state fidelities of two-qubit gates.

\begin{tabular}{cccc}
\hline \hline$\alpha$ & $\beta$ & Output state & Fidelity \\
\hline 0 & 0 & $\left|\psi_{1}\right\rangle=|H\rangle|+\rangle+|V\rangle|-\rangle$ & $0.831 \pm 0.033$ \\
0 & $\pi / 2$ & $\left|\psi_{2}\right\rangle=|H\rangle|R\rangle+|V\rangle|L\rangle$ & $0.847 \pm 0.036$ \\
0 & $\pi$ & $\left|\psi_{3}\right\rangle=|H\rangle|-\rangle+|V\rangle|+\rangle$ & $0.924 \pm 0.025$ \\
0 & $-\pi / 2$ & $\left|\psi_{4}\right\rangle=|H\rangle|L\rangle+|V\rangle|R\rangle$ & $0.899 \pm 0.028$ \\
$\pi$ & 0 & $\left|\psi_{5}\right\rangle=|H\rangle|+\rangle-|V\rangle|-\rangle$ & $0.912 \pm 0.028$ \\
$\pi$ & $\pi / 2$ & $\left|\psi_{6}\right\rangle=|H\rangle|R\rangle-|V\rangle|L\rangle$ & $0.913 \pm 0.028$ \\
$\pi$ & $\pi$ & $\left|\psi_{7}\right\rangle=|H\rangle|-\rangle-|V\rangle|+\rangle$ & $0.925 \pm 0.024$ \\
$\pi$ & $-\pi / 2$ & $\left|\psi_{8}\right\rangle=|H\rangle|L\rangle-|V\rangle|R\rangle$ & $0.910 \pm 0.027$ \\
\hline \hline
\end{tabular}

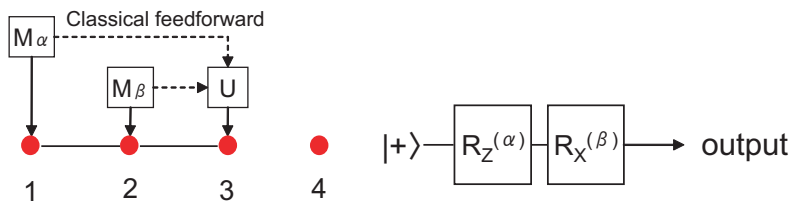

FIG. 5: Physical implementation and the quantum circuit of a single-qubit rotation.

statistical mixture of strategies does not improve the optimal fidelity, the possible strategies are exhausted by all the combinations of the grouping of eight states, which is finite, and we have exhaustively confirmed that the above number is the optimal one. On the other hand, average of the eight fidelities in Table $\Pi$ gives $0.895 \pm$ 0.010 , indicating that our demonstration of the one-way QC achieved the fidelity that is only possible through the contribution of the entanglement in the produced cluster state.

Single-qubit rotations. The quantum circuit in Fig. [5] shows a simple implementation of a single-qubit rotation. Qubit 4 is disentangled from the cluster state by measuring in the basis $\{|+\rangle,|-\rangle\}$. The input state is $\left|\psi_{\text {in }}\right\rangle=|+\rangle$. Qubits 1 and 2 are measured in the basis $B^{\prime}(\alpha)$ and $B(\beta)$, respectively, where $B^{\prime}(\theta)=\left\{\frac{|+\rangle+e^{-i \theta}|-\rangle}{\sqrt{2}}, \frac{|+\rangle-e^{-i \theta}|-\rangle}{\sqrt{2}}\right\}$. The outcomes are feedforwarded and Pauli operations are applied on qubits 3 accordingly, resulting in the output state $\left|\psi_{\text {out }}\right\rangle=R_{X}(\beta) R_{Z}(\alpha)|+\rangle$, where $R_{X}(\theta)=$ $\exp \left(-i \theta \sigma_{X} / 2\right)$. The gate instructions $(\alpha, \beta)$ are given to qubits 1 and 2 , and only two bits are communicated to qubit 3. In experiment, we chose 6 combinations of $(\alpha, \beta)$, and measured the fidelity of the output states. Table III shows the results. As in the case of two-qubit gates, we determine the upper bound on the average fidelity when we do not have entanglement at all. An optimal strategy is to divide the 6 states into 4 groups, e.g., (i) $|H\rangle$, (ii) $|V\rangle$, (iii) $|+\rangle,|R\rangle$, and (iv) $|-\rangle,|L\rangle$, and to send the identity of the group. Using this information, qubit 3 is prepared in one of the states (i) $|H\rangle$, (ii) $|V\rangle$, (iii) $|+\rangle+|R\rangle$, or (iv) $|-\rangle+|L\rangle$ (normalization factors
TABLE II: Output state fidelities of single-qubit rotations.

\begin{tabular}{cccc}
\hline \hline$\alpha$ & $\beta$ & Output state & Fidelity \\
\hline 0 & 0 & $|+\rangle$ & $0.944 \pm 0.022$ \\
$\pi$ & 0 & $|-\rangle$ & $0.888 \pm 0.029$ \\
$\pi / 2$ & 0 & $|R\rangle$ & $0.928 \pm 0.026$ \\
$-\pi / 2$ & 0 & $|L\rangle$ & $0.969 \pm 0.017$ \\
$\pi / 2$ & $\pi / 2$ & $|H\rangle$ & $0.915 \pm 0.029$ \\
$\pi / 2$ & $-\pi / 2$ & $|V\rangle$ & $0.917 \pm 0.027$ \\
\hline \hline
\end{tabular}

omitted). This strategy gives the average fidelity of the 6 states $(2 / 6) \times 1+(4 / 6) \times \cos ^{2}(\pi / 8) \approx 0.902$. On the other hand, average of the six fidelities in Table II gives $0.926 \pm 0.010$, indicating that the entanglement in the produced state really contributes to the one-way QC.

We have demonstrated a high-fidelity four-photon cluster state that is distinguished from other types of genuine four-qubit entanglement such as GHZ, $W$, and Dicke states. We have also shown that the results of the basic operations of one-way QC surpass the classical bounds, which indicates that the entanglement of cluster states really contributes to one-way QC. The model of oneway $\mathrm{QC}$ is unique in that the computation process is divided into preparation of a nonlocal static resource (a cluster state) and dynamic execution involving only local measurements and classical communication. This gives a close link to the quantum communication problems, and various classical bounds related to communication tasks such as the proposed bound here, which may be called "classical RSP bound", will be used as benchmarks toward the realization of quantum computing. The relation between such bounds and the computational power is also an interesting problem, which may give us a deeper insight into the role of entanglement in the quantum computation.

We thank Şahin K. Özdemir, Ryo Namiki, Shigeki Takeuchi, Ryo Okamoto, and Tomohisa Nagata, for their helpful advice and discussions. This work was supported by 21st Century COE Program by the Japan Society for the Promotion of Science and a MEXT Grant-in-Aid for Young Scientists (B) 17740265.

* tokunaga.yuuki@lab.ntt.co.jp

[1] R. Raussendorf and H. J. Briegel, Phys. Rev. Lett. 86, 5188 (2001).

[2] P. Walther et al. , Nature 434, 169 (2005).

[3] N. Kiesel et al., Phys. Rev. Lett. 95, 210502 (2005).

[4] P. Walther et al., Phys. Rev. Lett. 95, 020403 (2005).

[5] R. Prevedel et al., Nature 445, 65 (2007).

[6] C.-Y. Lu et al., Nature Physics 3, 91 (2007).

[7] G. Vallone et al., Phys. Rev. Lett. 98, 180502 (2007). 
[8] K. Chen et al., Phys. Rev. Lett. 99, 120503 (2007).

[9] Y. Tokunaga et al., Phys. Rev. A 74, 020301(R) (2006).

[10] Y. Tokunaga et al., Phys. Rev. A 71, 030301(R) (2005).

[11] D. Gottesman and I. L. Chuang, Nature 402, 390 (1999).

[12] P. G. Kwiat et al., Phys. Rev. A 60, R773 (1999).

[13] G. Tóth and O. Gühne, Phys. Rev. Lett. 94, 060501
(2005).

[14] G. Tóth and O. Gühne, Phys. Rev. A 72, 022340 (2005).

[15] A. Sanpera et al., Phys. Rev. A 63, 050301(R) (2001).

[16] C. H. Bennett et al., Phys. Rev. Lett. 87, 077902 (2001). 